\title{
Calculation of AVA Responses for Finely Layered Reservoirs
}

\author{
Ying An $(\mathbb{D})$ and Jun Lu $(\mathbb{D}$ \\ Key Laboratory of Marine Reservoir Evolution and Hydrocarbon Accumulation Mechanism, Ministry of Education, \\ China University of Geosciences, Beijing 100083, China \\ Correspondence should be addressed to Jun Lu; lujun615@163.com
}

Received 11 December 2017; Revised 30 March 2018; Accepted 17 April 2018; Published 22 May 2018

Academic Editor: Andrés Sáez

Copyright (C) 2018 Ying An and Jun Lu. This is an open access article distributed under the Creative Commons Attribution License, which permits unrestricted use, distribution, and reproduction in any medium, provided the original work is properly cited.

In oil and gas seismic exploration, responses of amplitude variation with incidence angle (AVA) of strata with a single thin layer have been extensively studied, thus laying the theoretical foundation for the prediction of finely layered reservoirs. However, studies on AVO responses of common shale-sand finely layered reservoirs are rare because of mathematical modeling problems involved with such complicated reservoirs. To accurately analyze the AVA responses of finely layered reservoirs, we describe such a reservoir as a transversely isotropic medium with a vertical axis of symmetry (VTI medium). We calculate parameters of the VTI medium according to the thickness ratios of gas-saturated sand layers in finely layered reservoirs and investigate the characteristics of AVA curves and gathers. The results show that the anisotropy of finely layered reservoirs will lead to large errors in actual seismic response recognition for large incidence angles or total thicknesses of sand layers close to half of the entire reservoir. The simultaneous use of $P P$ - and $P S$-waves in AVA response analysis can provide better estimates of the thickness ratios of gas-saturated sand layers to finely layered reservoirs.

\section{Introduction}

With the intensification of oil and gas exploration and development, shale-sand finely layered reservoirs have become important targets. However, studies on finely layered reservoirs have been lacking owing to the small thickness and complex internal structure. In particular, seismic methods have seldom been considered in the study of the interlayer structure, net-to-gross $(N / G)$, and lithology. In a seismic exploration, when the length of seismic waves is much longer than the thickness of finely layered reservoir, the mathematical model of a finely layered reservoir can be considered as a VTI medium (transversely isotropic medium with a vertical symmetry axis), a type of effective anisotropic medium [1-4]. Stovas and Ursin [5] proved that, for small values of reflection coefficients, the transition between effective medium and time-average medium occurs around a ratio of wavelength to layer thickness equal to 4. Bruggeman [6] proposed a mathematical method for deriving the time-average elastic constants of layered solids. He established the theoretical basis for the study of the anisotropic seismic responses of periodic finely layered reservoirs by describing the properties of an average solid with axial symmetry and five elastic constants. Krey and Helbig [7] studied the propagation law of reflected waves in an anisotropic medium and proposed that the combination of sand and shale layers for the finely layered reservoir can lead to the seismic anisotropy theoretically. Such finely layered reservoirs are characterized by an interbedded structure of shale and sand, which can be described by the $N / G$ ratio. Here, $N$ denotes the thickness of sand layers and $G$ denotes the total thickness of finely layered reservoirs. Vernik et al. [8] used $P$ - and $S$-impedance to estimate the $N / G$ ratios. Stovas et al. [9] studied the relationships among the $N / G$ ratio, anisotropy of a finely layered reservoir, and seismic responses, which are the bases of our research.

AVA (or AVO: Amplitude Variation with Offset) technology can be directly applied to oil and gas prediction according to the lithology and fluid information contained in the variation law of the amplitude versus angle or offset of the reservoirs. However, the traditional AVA theory is based on the assumption of isotropic media, which will lead to large errors when applied to anisotropic media. Schoenberg [10] presented that transversely isotropic media (TI) are equivalent to periodic deposition under the condition of long-wavelength assumption. Banik [11] used Thomsen [12] 
parameters to describe the $P P$-wave reflection coefficient equation of transversely isotropic media. Rüger $[13,14]$ proposed the $P P$ - and $P S$-wave reflection coefficient equations of both VTI and HTI (transversely isotropic medium with a horizontal symmetry axis) media. These studies considered the interbedded structure of shale-sand inside a finely layered reservoir as a whole, which reflects VTI characteristics, without focusing on single thin-bed reflection.

Liu and Schmitt [15] deduced the exact reflection coefficient equations in the acoustic domain and simulated the AVA response characteristics of a thin bed. Pan and Innanen [16] analyzed the relationship between AVA effects and frequency based on the reflection coefficient spectrum theory of a thin elastic medium. Yang et al. [17] proposed the simplified reflection and transmission coefficients for a single thin layer. However, only few studies have focused on the AVA responses of the more common sedimentary structure of shale-sand finely layered reservoir owing to the complex combination relationship and various internal structures. Therefore, it is of great significance to study the AVA response characteristics of shale-sand finely layered reservoirs.

In this work, based on the theory of long-wavelength anisotropy, we consider a finely layered reservoir as a VTI medium model. The model parameters are calculated according to the thickness ratios of gas-saturated sand layers and a finely layered reservoir. Using the reflection coefficient equations proposed by Rüger [13], we simulated and analyzed the $P P$ - and $P S$-wave AVA responses of different models in terms of AVA curves and gathers. Eventually, based on Stovas et al.' model, $P P$ - and $P S$-wave AVA gathers are derived using both the isotropic and anisotropic reflection coefficient equations, respectively. We verified the reliability of our method through comparative analysis of our simulation results with those of Stovas et al. [9].

\section{Methodology}

In this section, we propose the principles and method of studying the anisotropic characteristics of a finely layered reservoir and introduce the scheme of constructing our proposed model.

2.1. N/G Ratios and Reflection Coefficient Equations. The VTI medium is a transversely isotropic medium with a vertical axis of symmetry, as shown in Figure 1. Then, the stiffness matrix of the VTI medium can be written as

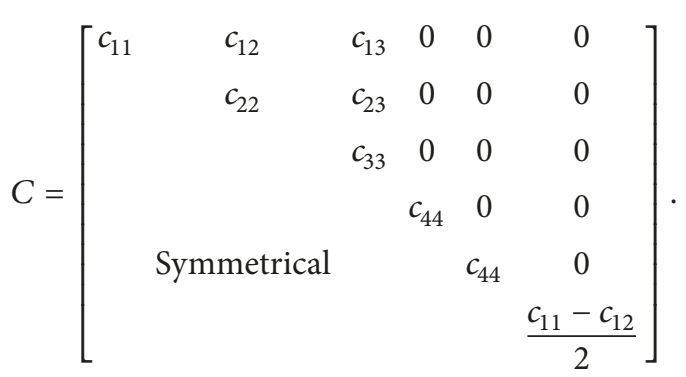

The parameters $c_{33, a}, c_{44, a}, \rho_{a}$ (density), $V_{P a}$ ( $P$-wave velocity), and $V_{S a}$ ( $S$-wave velocity) are for shale layers, and the parameters $c_{33, b}, c_{44, b}, \rho_{b}, V_{P b}$, and $V_{S b}$ are for sand layers; the subscripts " $a$ " and " $b$ " denote the corresponding parameters for the shale and sand layers, respectively. The relationship between the elastic parameters $c_{i j}$ of the stiffness matrix of the VTI medium and $N / G$ ratio can be expressed as [9]

$$
\begin{aligned}
& c_{11}=c_{33, a} \frac{1+4 \xi_{a}^{2}(N / G)[1-(N / G)]\left\{\Delta c_{44}\left[\Delta c_{33}\left(1-\Delta c_{44} / 2\right)-\xi_{a}^{2} \Delta c_{44}\left(1-\Delta c_{33} / 2\right)\right] /\left[\left(1-\Delta c_{44} / 2\right)^{2}\left(1+\Delta c_{33} / 2\right)\right]\right\}}{1-(N / G)\left[\Delta c_{33} /\left(1+\Delta c_{33} / 2\right)\right]} \\
& c_{13}=c_{33, a} \frac{1-2 \xi_{a}^{2}+2 \xi_{a}^{2}(N / G)\left\{\left(\Delta c_{33}-\Delta c_{44}\right) /\left[\left(1-\Delta c_{44} / 2\right)\left(1+\Delta c_{33} / 2\right)\right]\right\}}{1-(N / G)\left[\Delta c_{33} /\left(1+\Delta c_{33} / 2\right)\right]} \\
& c_{33}=c_{33, a} \frac{1}{1-(N / G)\left[\Delta c_{33} /\left(1+\Delta c_{33} / 2\right)\right]}, \\
& c_{44}=c_{44, a} \frac{1}{1-(N / G)\left[\Delta c_{44} /\left(1+\Delta c_{44} / 2\right)\right]}
\end{aligned}
$$

where $\xi_{a}^{2}=c_{44, a} / c_{33, a}$, which is equal to $V_{S a}^{2} / V_{P a}^{2}$. The density $(\rho)$ of the VTI medium can be expressed as

$$
\rho=\rho_{a}\left[1+\left(\frac{N}{G}\right) \frac{\Delta \rho}{1-\Delta \rho / 2}\right],
$$

the contrasts of the stiffness coefficients and densities between shale and sand layers in the VTI medium are

$$
\Delta c_{33}=2 \frac{c_{33, b}-c_{33, a}}{c_{33, b}+c_{33, a}}
$$

$$
\begin{aligned}
\Delta c_{44} & =2 \frac{c_{44, b}-c_{44, a}}{c_{44, b}+c_{44, a}}, \\
\Delta \rho & =2 \frac{\rho_{b}-\rho_{a}}{\rho_{b}+\rho_{a}} .
\end{aligned}
$$

The properties of the elastic medium are determined by the stiffness matrix $C$, which establishes the relationship between stress and strain. However, the physical meaning of the coefficients of the elastic wave equation expressed by the 


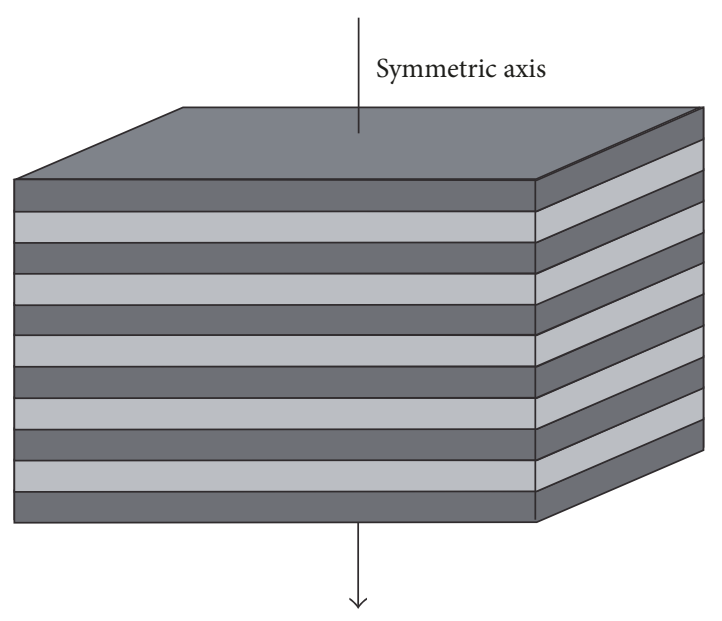

FIGURE 1: Schematic diagram of the VTI media.

stiffness matrix $C$ is complex and not intuitionistic. Thomsen [12] used $P$ - and $S$-wave vertical velocities $\left(V_{P 0}\right.$ and $\left.V_{S 0}\right)$ and anisotropic parameters $(\varepsilon$ and $\delta$ ) to describe the anisotropy of the VTI medium, which can be calculated by the stiffness coefficients as

$$
\begin{aligned}
V_{P 0} & =\sqrt{\frac{c_{33}}{\rho}}, \\
V_{S 0} & =\sqrt{\frac{c_{44}}{\rho}}, \\
\varepsilon & =\frac{c_{11}-c_{33}}{2 c_{33}}, \\
\delta & =\frac{\left(c_{13}+c_{44}\right)^{2}-\left(c_{33}-c_{44}\right)^{2}}{2 c_{33}\left(c_{33}-c_{44}\right)},
\end{aligned}
$$

$\varepsilon$ denotes the fractional difference between the horizontal and vertical $P$-wave velocities squared; $\delta$ describes the variation of $P$-wave velocity with phase angle for the near vertical propagation [13].

The anisotropy parameters can be related to the combination of shale and sand layers according to the theories proposed by Stovas et al. [9] by substituting (2) into (5):

$$
\begin{aligned}
& \varepsilon=2 \xi_{a}^{2}\left(\frac{N}{G}\right)\left[1-\left(\frac{N}{G}\right)\right] \Delta c_{44} \chi \\
& \delta \approx\left(\frac{N}{G}\right) \xi_{a}^{2}\left[1-2 \xi_{a}^{2}\left(\frac{N}{G}\right)\right] \Delta c_{44} \psi,
\end{aligned}
$$

where the contrast constants $\chi$ and $\psi$ are given by

$$
\begin{aligned}
& \chi=\frac{\Delta c_{33}\left(1-\Delta c_{44} / 2\right)-\xi_{a}^{2} \Delta c_{44}\left(1-\Delta c_{33} / 2\right)}{\left(1-\Delta c_{44} / 2\right)^{2}\left(1+\Delta c_{33} / 2\right)}, \\
& \psi=\frac{\left(\Delta c_{33}-\Delta c_{44}\right)}{\left(1-\Delta c_{44}^{2} / 2\right)\left(1+\Delta c_{33} / 2\right)} .
\end{aligned}
$$

Then, we combine two sets of the finely layered reservoirs into a model containing two VTI medium layers, which produce a reflection interface. The parameters of the upper (above the interface) and lower (below the interface) VTI media are indicated by the subscripts "1" and " 2 ," respectively. At the interface, the Zoeppritz equation completely describes the relationship between the plane wave reflection coefficients and incidence angles [18]. However, this equation is too complex for the AVA analysis of finely layered reservoirs. Therefore, we adopted Rüger's [13] approximate solutions of the Zoeppritz equation for the VTI media as follows:

$$
\begin{aligned}
& R_{P P}=\frac{1}{2} \frac{\Delta Z}{\bar{Z}}+\frac{1}{2}\left[\frac{\Delta V_{P 0}}{\bar{V}_{P 0}}-\left(\frac{2 \bar{V}_{S 0}}{\bar{V}_{P 0}}\right)^{2} \frac{\Delta G}{\bar{G}}\right] \sin ^{2} i+\frac{1}{2} \\
& \text { - } \frac{\Delta V_{P 0}}{\bar{V}_{P 0}} \sin ^{2} i \tan ^{2} i+\frac{1}{2}\left(\delta_{2}-\delta_{1}\right) \sin ^{2} i+\frac{1}{2}\left(\varepsilon_{2}-\varepsilon_{1}\right) \\
& \text { - } \sin ^{2} i \tan ^{2} i \text {, } \\
& R_{P S}=-\frac{1}{2} \frac{\Delta \rho}{\bar{\rho}} \frac{\sin i}{\cos j}-\frac{\bar{V}_{S 0}}{\bar{V}_{P 0}}\left(\frac{\Delta \rho}{\bar{\rho}}+2 \frac{\Delta V_{S 0}}{\bar{V}_{S 0}}\right) \sin i \cos i \\
& +\left(\frac{\bar{V}_{S 0}}{\bar{V}_{P 0}}\right)^{2}\left(\frac{\Delta \rho}{\bar{\rho}}+2 \frac{\Delta V_{S 0}}{\bar{V}_{S 0}}\right) \frac{\sin ^{3} i}{\cos j} \\
& +\left\{\left[\frac{\bar{V}_{P 0}^{2}}{2\left(\bar{V}_{P 0}^{2}-\bar{V}_{S 0}^{2}\right) \cos j}-\frac{\bar{V}_{P 0} \bar{V}_{S 0} \cos i}{2\left(\bar{V}_{P 0}^{2}-\bar{V}_{S 0}^{2}\right)}\right]\right. \\
& \left.\cdot\left(\delta_{2}-\delta_{1}\right)\right\} \sin i \\
& +\left[\frac{\bar{V}_{S 0} \bar{V}_{P 0} \cos i}{\left(\bar{V}_{P 0}^{2}-\bar{V}_{S 0}^{2}\right)}\left(\delta_{2}-\delta_{1}+\varepsilon_{1}-\varepsilon_{2}\right)\right] \sin ^{3} i \\
& -\left[\frac{\bar{V}_{P 0}^{2}}{\left(\bar{V}_{P 0}^{2}-\bar{V}_{S 0}^{2}\right) \cos j}\left(\delta_{2}-\delta_{1}+\varepsilon_{1}-\varepsilon_{2}\right)\right] \sin ^{3} i \\
& +\left[\frac{\bar{V}_{S 0}^{2}}{2\left(\bar{V}_{P 0}^{2}-\bar{V}_{S 0}^{2}\right) \cos j}\left(\delta_{1}-\delta_{2}\right)\right] \sin ^{3} i \\
& +\left[\frac{\bar{V}_{S 0}^{2}}{\left(\bar{V}_{P 0}^{2}-\bar{V}_{S 0}^{2}\right) \cos j}\left(\delta_{2}-\delta_{1}+\varepsilon_{1}-\varepsilon_{2}\right)\right] \sin ^{5} i,
\end{aligned}
$$

where

$$
\begin{aligned}
\bar{V}_{P 0} & =\frac{1}{2}\left(V_{P 0_{1}}+V_{P 0_{2}}\right), \\
\Delta V_{P 0} & =V_{{P 0_{2}}_{2}}-V_{P 0_{1}}, \\
\bar{V}_{S 0} & =\frac{1}{2}\left(V_{S 0_{1}}+V_{S 0_{2}}\right), \\
\Delta V_{S 0} & =V_{S_{0_{2}}}-V_{S 0_{1}},
\end{aligned}
$$




$$
\begin{aligned}
\bar{\rho} & =\frac{1}{2}\left(\rho_{1}+\rho_{2}\right), \\
\Delta \rho & =\rho_{2}-\rho_{1} .
\end{aligned}
$$

$i$ and $j$ denote the incidence and transmission phase angles of the $P$-wave, respectively. The vertical $P$-wave impedance $Z \equiv \rho V_{P 0}$ and the vertical shear modulus $G \equiv \rho V_{S 0}{ }^{2}$. follows:

Equation (8) consists of isotropic and anisotropic parts as

$$
\begin{aligned}
& R_{P P}=R_{P P}^{\text {iso }}+R_{P P}^{\text {anis }}, \\
& R_{P S}=R_{P S}^{\text {iso }}+R_{P S}^{\text {anis }},
\end{aligned}
$$

where the superscripts "iso" and "anis" denote the isotropic and anisotropic parts of the $P P$ reflection coefficient $\left(R_{P P}\right)$ or $P S$ reflection coefficient $\left(R_{P S}\right)$, respectively. In (8), items with anisotropic parameters belong to the anisotropic part and other items belong to the isotropic part. When the anisotropy parameters are zero, the anisotropic part will be zero. Then, (8) will be degenerated into the reflection coefficient equations of the isotropic media. If the parameters of the shale and sand layers and the corresponding $N / G$ ratios are known, the velocity, density, and anisotropy parameters of the finely layered reservoir can be calculated according to (2)-(6). Then, the reflection coefficients and AVA gathers can be obtained using (8).

2.2. Biot-Gassmann Fluid Model. In building our model, we consider the existence of fluid in the sand layers of the finely layered reservoir. In general, the Biot-Gassmann equations [19] are used to calculate seismic velocities for fluid-filled porous media. The volume moduli of a known rock matrix, skeleton, and pore fluid are used to calculate the elastic moduli of a porous medium with fluid saturation. The $P$ - and $S$-wave velocities and density of the fluid-saturated sand layer can be calculated according to the following equations:

$$
\begin{aligned}
& V_{P b}^{\text {sat }}=\sqrt{\frac{K_{b}^{\text {sat }}+(4 / 3) \mu_{b}^{\text {sat }}}{\rho_{b}^{\text {sat }}}}, \\
& V_{S b}^{\text {sat }}=\sqrt{\frac{\mu_{b}^{\text {sat }}}{\rho_{b}^{\text {sat }}}}
\end{aligned}
$$

where $\rho_{b}^{\text {sat }}, K_{b}^{\text {sat }}$, and $\mu_{b}^{\text {sat }}$ denote the density, bulk modulus, and shear modulus of the fluid-saturated sand layer, respectively. The density of the sand layer is given as follows:

$$
\begin{aligned}
\rho_{b}^{\text {sat }} & =(1-\phi) \rho_{m}+\phi \rho_{f}, \\
\rho_{f} & =S_{w} \rho_{w}+S_{o} \rho_{o}+S_{g} \rho_{g},
\end{aligned}
$$

where $S_{o}, S_{g}$, and $S_{w}$ denote the saturation of oil, gas, and water, respectively; $\rho_{o}, \rho_{g}$, and $\rho_{w}$ denote the density of oil, gas, and water, respectively; $\phi$ is the porosity; and $\rho_{m}$ denotes the grain density of the sand layer. The shear modulus $\mu_{b}^{\text {sat }}$
TABLE 1: Physical parameters of the fluid.

\begin{tabular}{lcc}
\hline Fluid & Bulk modulus $(\mathrm{GPa})$ & Density $\left(\mathrm{g} / \mathrm{cm}^{3}\right)$ \\
\hline Gas & 0.02 & 0.14 \\
\hline
\end{tabular}

of the fluid-saturated sand layer is not affected by the fluid, which is equal to that of the dry rock $\mu_{d}$ :

$$
\mu_{b}^{\text {sat }}=\mu_{d}
$$

The bulk modulus of the sand layer $K_{b}^{\text {sat }}$ can be calculated as follows:

$$
K_{b}^{\mathrm{sat}}=K_{d}+\frac{\left(1-K_{d} / K_{m}\right)^{2}}{\phi / K_{f}+(1-\phi) / K_{m}-K_{d} / K_{m}^{2}},
$$

where $K_{d}, K_{m}$, and $K_{f}$ denote the bulk modulus of the grain, matrix, and pore fluid, respectively. The bulk modulus of the pore fluid can be calculated by

$$
\frac{1}{K_{f}}=\frac{S_{w}}{K_{w}}+\frac{S_{o}}{K_{o}}+\frac{S_{g}}{K_{g}}
$$

Through (11)-(15), we can obtain the $P$ - and $S$-wave velocities and density of the sand layers of a finely layered reservoir under the condition of arbitrary fluid and porosity.

\section{Simulation of a Finely Layered Reservoir}

We build a mathematical finely layered reservoir model, in which the shale and gas-saturated sand layers are interbedded and sandwiched within two semifinite shale layers, as shown in Figure 2(a). The interbedded shale and gas-saturated sand layers are equivalent to a VTI medium in our approach, as shown in Figure 2(b). In this section, we calculate the parameters of the VTI medium and simulate the AVA curves and gathers with different $N / G$ ratios.

Assuming that the reservoir is pure quartz sandstone and the only fluid in the pores is gas, we build the theoretical finely layered reservoir model according to the flows shown in Figure 3. First, we calculate the bulk modulus of the pore fluid $K_{f}=0.02 \mathrm{GPa}$ according to Table 1 and (15). Second, we calculate the density of the gas-saturated sand layers $\rho_{b}^{\text {sat }}$ $=1.82 \mathrm{~g} / \mathrm{cm}^{3}$ according to (12). Then, the velocities of gassaturated sand layers are obtained using (11), (13), and (14) based on the parameters in Table $2: V_{P b}^{\text {sat }}=2085 \mathrm{~m} / \mathrm{s}, V_{S b}^{\text {sat }}$ $=1365 \mathrm{~m} / \mathrm{s}$. $N$ and $G$ indicate the total thickness of gassaturated sand layers and finely layered reservoir, respectively. The parameters of the shale layers in the model are $V_{\mathrm{Pa}}=$ $2300 \mathrm{~m} / \mathrm{s}, V_{S a}=850 \mathrm{~m} / \mathrm{s}, \rho_{a}=2.0 \mathrm{~g} / \mathrm{cm}^{3}$. In this manner, mathematical models for a finely layered reservoir with different $N / G$ ratios can be built.

3.1. Model Parameters with Different N/G Ratios. Given a specific $N / G$ ratio, we can calculate the stiffness coefficient $c_{i j}$ according to (2) and then obtain the values of $V_{P 0}, V_{S 0}$, $\rho, \varepsilon$, and $\delta$ of the VTI medium using (3) and (5). Model parameters of finely layered reservoirs with different $N / G$ 


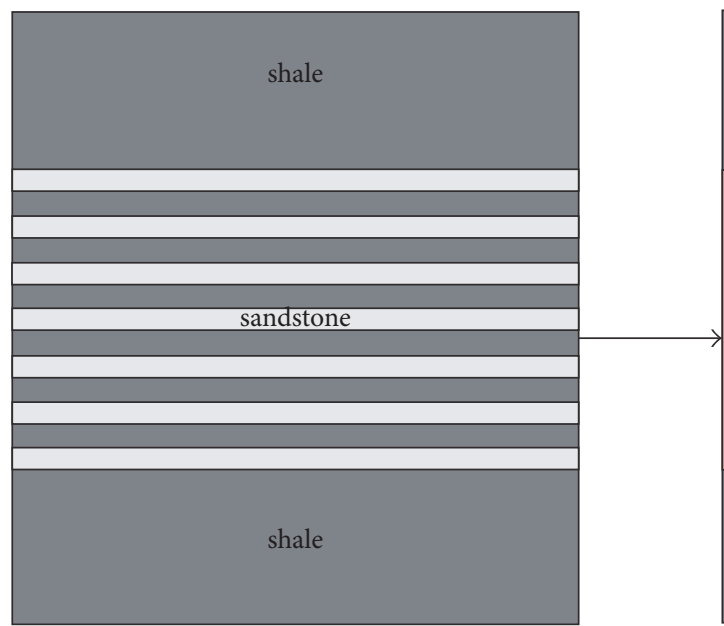

(a)

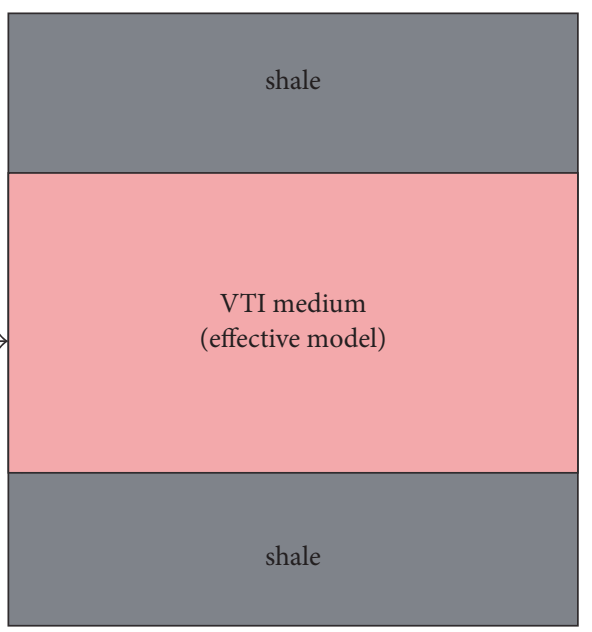

(b)

FIGURE 2: Mathematical models of the finely layered reservoir: (a) model with the interbedded shale-sand structure; (b) model which shalesand interbeds equivalently to a VTI medium.

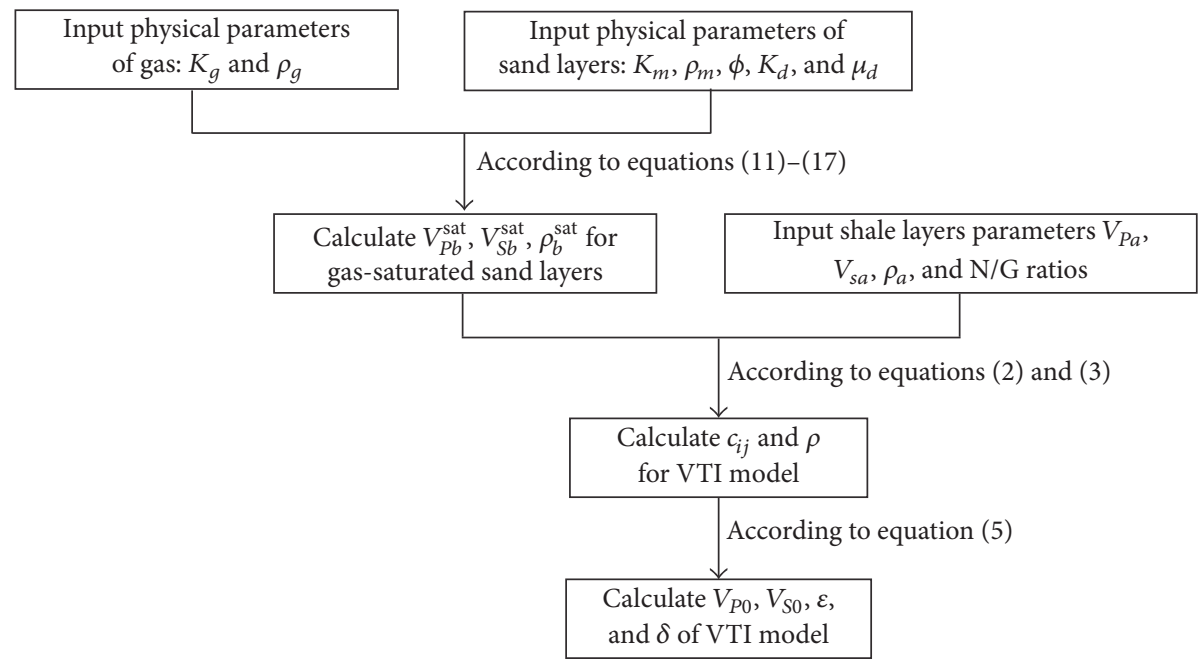

FIGURE 3: Flow chart of model parameters with different $N / G$ ratios.

TABLE 2: Physical parameters of gas-saturated sand layers.

\begin{tabular}{lc}
\hline Parameter name & Values \\
\hline Bulk modulus of grain $K_{m}(\mathrm{GPa})$ & 40 \\
Density of grain $\rho_{m}\left(\mathrm{~g} / \mathrm{cm}^{3}\right)$ & 2.65 \\
Porosity $\phi$ & $33 \%$ \\
Gas saturation $S_{w}$ & 1.0 \\
Bulk modulus of grain $K_{d}(\mathrm{GPa})$ & 3.34 \\
Shear modulus of grain $\mu_{d}(\mathrm{GPa})$ & 3.40 \\
\hline
\end{tabular}

ratios are shown in Table 3 and Figure 4. Figures 4(a), 4(b), and $4(\mathrm{c})$ show that the thickness of gas-saturated sand layers in the finely layered reservoir becomes larger with increasing $N / G$ ratios. Therefore, the property of the finely layered reservoir closely reflects gas-saturated sand layers; both
TABLE 3: Model parameters of the finely layered reservoirs.

\begin{tabular}{lccccc}
\hline$N / G$ & $V_{P 0}(\mathrm{~m} / \mathrm{s})$ & $V_{S 0}(\mathrm{~m} / \mathrm{s})$ & $\rho\left(\mathrm{g} / \mathrm{cm}^{3}\right)$ & $\varepsilon$ & $\delta$ \\
\hline 0.1 & 2272 & 879 & 1.982 & -0.0193 & -0.0314 \\
0.2 & 2246 & 912 & 1.964 & -0.0343 & -0.0584 \\
0.3 & 2222 & 947 & 1.946 & -0.0451 & -0.0806 \\
0.4 & 2199 & 986 & 1.928 & -0.0515 & -0.0976 \\
0.5 & 2177 & 1030 & 1.910 & -0.0536 & -0.1086 \\
0.6 & 2157 & 1079 & 1.892 & -0.0515 & -0.1126 \\
0.7 & 2137 & 1135 & 1.874 & -0.0451 & -0.1080 \\
0.8 & 2119 & 1200 & 1.856 & -0.0343 & -0.0919 \\
0.9 & 2101 & 1275 & 1.838 & -0.0193 & -0.0593 \\
\hline
\end{tabular}

$P$-wave velocities and densities decrease, while $S$-wave velocities increase with increasing $N / G$ ratios. Furthermore, the 


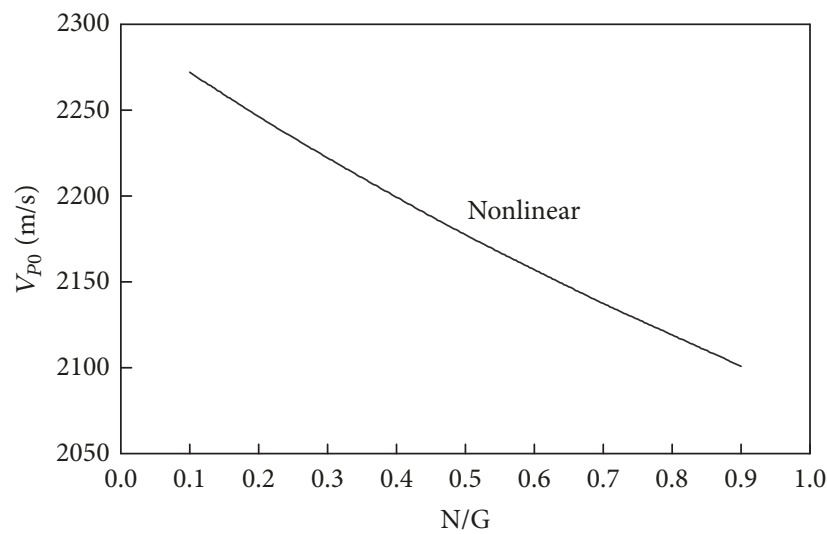

(a)

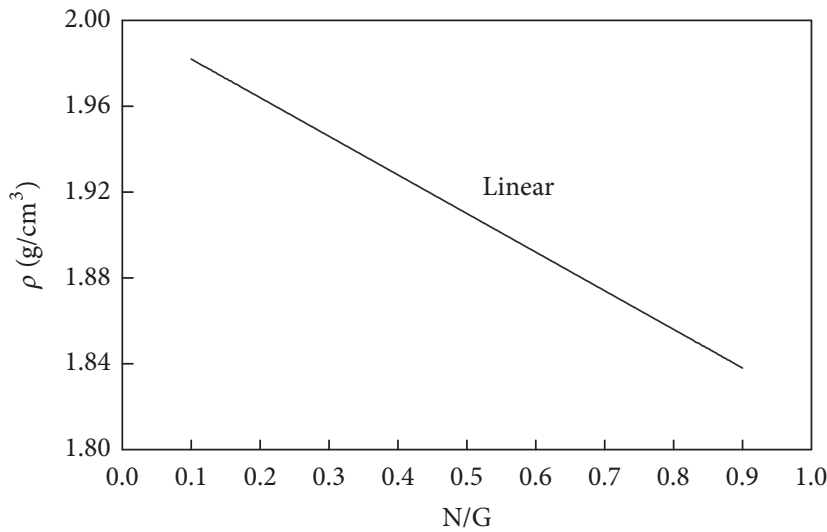

(c)

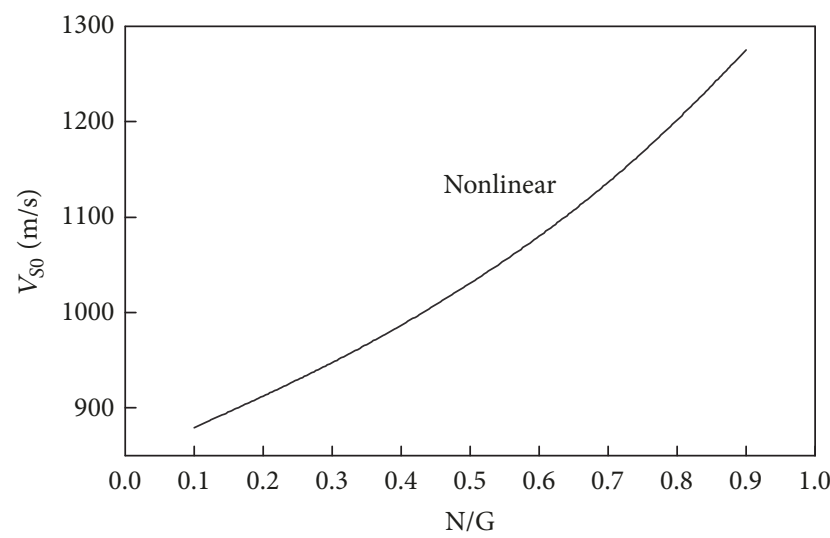

(b)

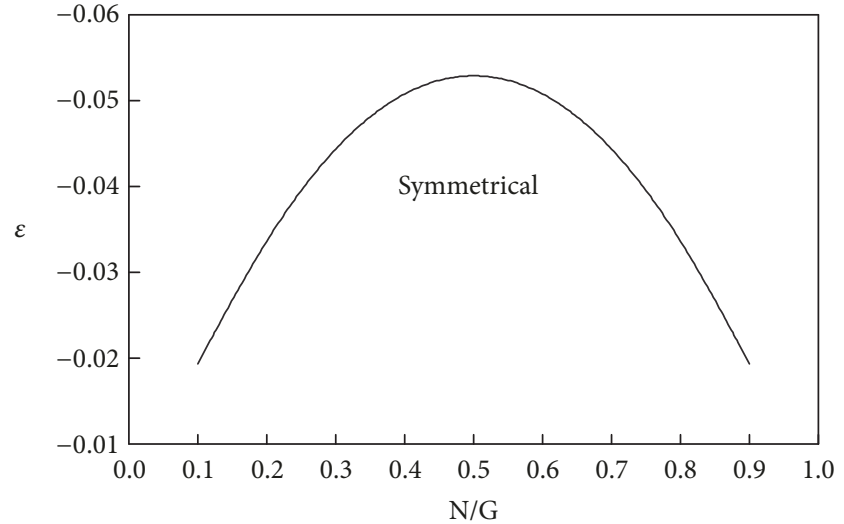

(d)

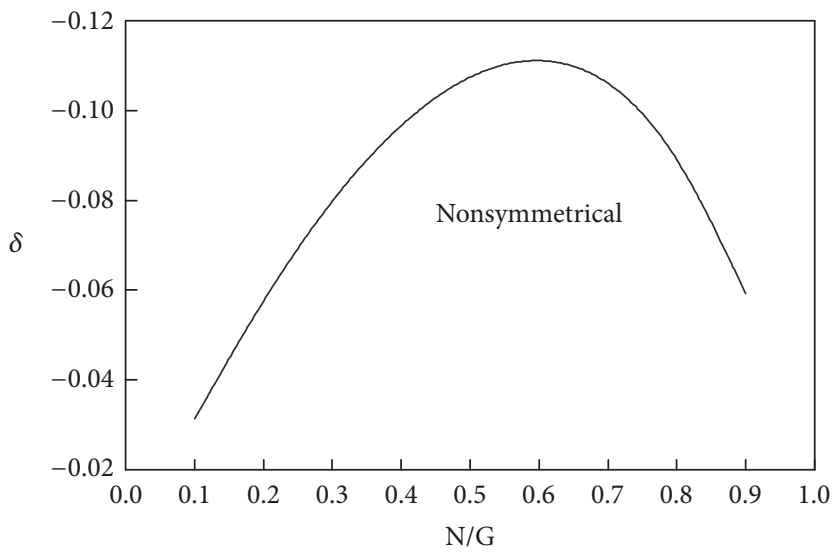

(e)

FIGURE 4: Changes in parameters with different $N / G$ ratios of the finely layered reservoirs: (a) $V_{P 0}$, (b) $V_{S 0}$, (c) $\rho$, (d) $\varepsilon$, and (e) $\delta$.

curves of $P$ - and $S$-waves are nonlinear, whereas the curve of density is linear. In Figure $4(\mathrm{~d})$, the $\varepsilon$ curve is symmetrical with variations in $N / G$ ratios. When $N / G=0.5$, the absolute value of $\varepsilon$ reaches the maximum and the $P$-wave anisotropy is the strongest. In Figure $4(\mathrm{e})$, when $N / G=0.6$, the absolute value of $\delta$ reaches the maximum, indicating the largest relative difference between the anisotropies of the $P$ - and $S V$ waves.
3.2. AVA Curves. According to the model parameters in Table 3, reflection coefficient curves of the $P P$ - and $P S$ waves (Figure 5) and the effect of anisotropy on the reflection coefficients of the $P P$ - and $P S$-waves (Figure 6) can be obtained using (8). In Figure 5(a), the reflection coefficients of the $P P$-wave are negative, and their absolute values increase with increasing incidence angles. At a constant incidence angle, the absolute value of the reflection 


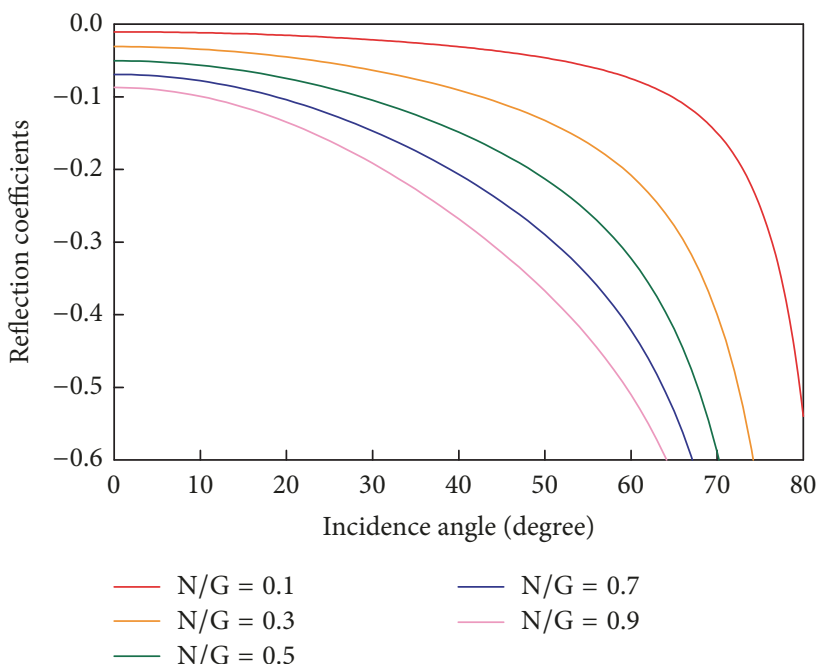

(a)

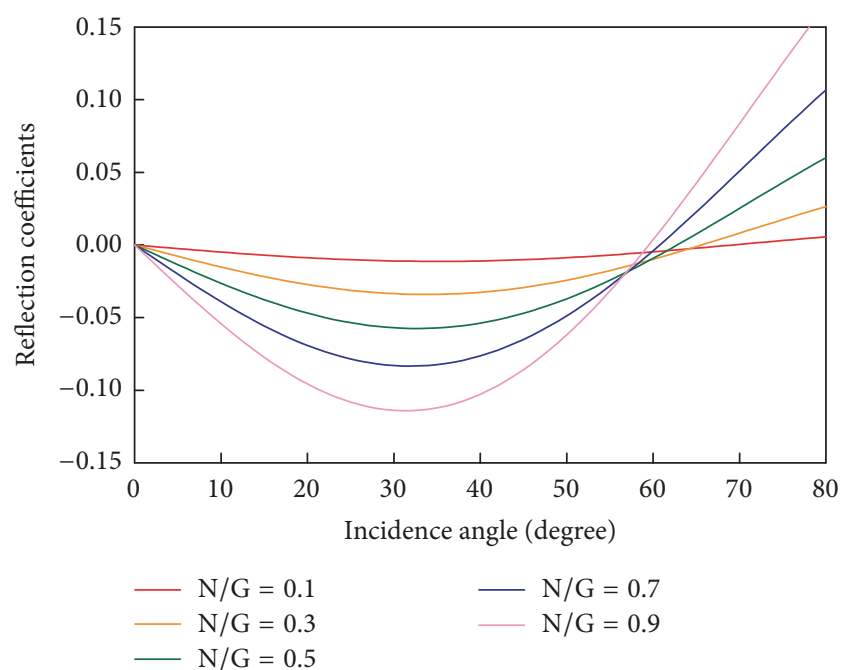

(b)

Figure 5: AVA curves of different $N / G$ models: (a) $P P$-wave and (b) $P S$-wave.

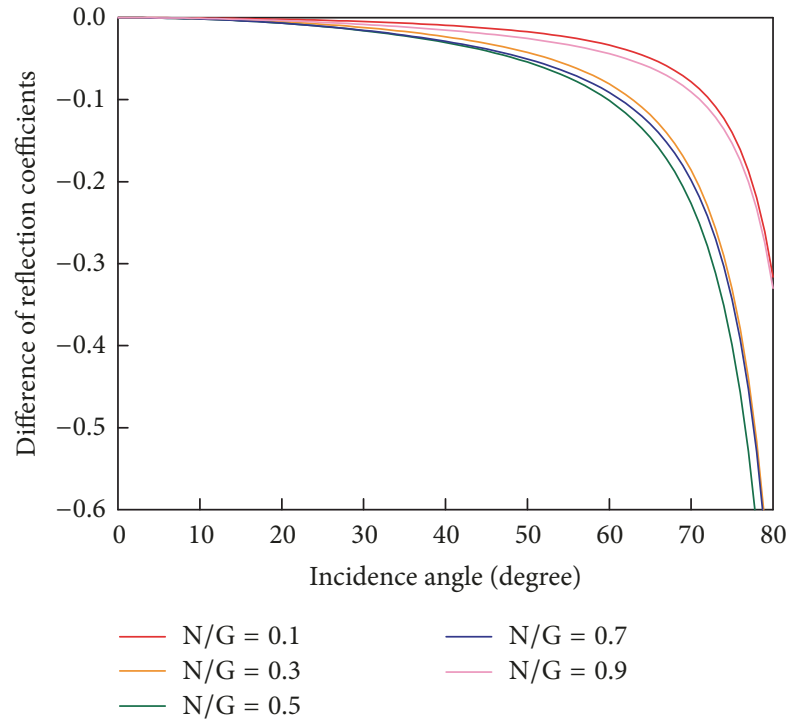

(a)

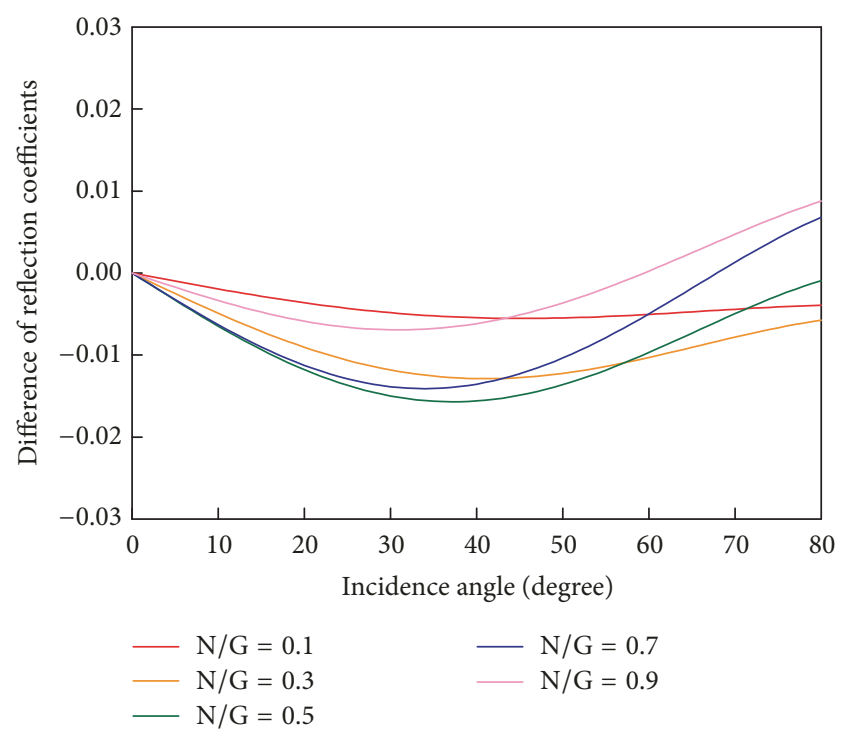

(b)

FIGURE 6: Differences in reflection coefficients between the VTI models with different $N / G$ ratios and the isotropic models: (a) $P P$-waves and (b) PS-waves.

coefficients increases with increasing $N / G$ ratios. The $P S$ wave reflection coefficients are negative at incidence angles of approximately $0-60^{\circ}$ in Figure 5(b). The minimum negative $P S$-wave reflection coefficients occur near the incidence angle of $30^{\circ}$. The reflection coefficients become positive at incidence angles over $60^{\circ}$. At a constant incidence angle, the absolute values of the reflection coefficients of both the $P P$ - and $P S$-waves increase with increasing $N / G$ ratios.

In order to study the influence of anisotropy on the reflection coefficients of the $P P$ - and $P S$-waves, we compare the AVA responses of the VTI models with those of models in which anisotropy parameters are set to zero. In Figure 6, the vertical coordinates show the difference between the reflection coefficients of the VTI model and the isotropic model, which reflects the influence of anisotropy on the reflection coefficients of the finely layered reservoir. In Figure 6(a), at incidence angles of $0-15^{\circ}$, differences in the reflection coefficients are close to 0 , implying that anisotropy has almost no effect on the reflection coefficients of the $P P$ wave. At incidence angles of $15-60^{\circ}$, differences between the reflection coefficients increase slightly with increasing incidence angles, implying that anisotropy has little influence on the coefficients. At incidence angles of $60-80^{\circ}$, the difference 


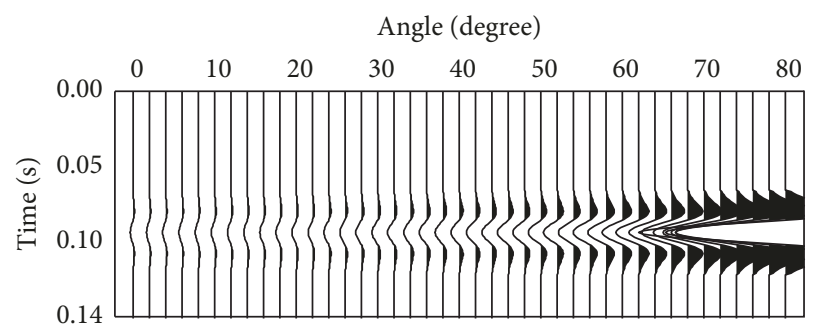

(a)

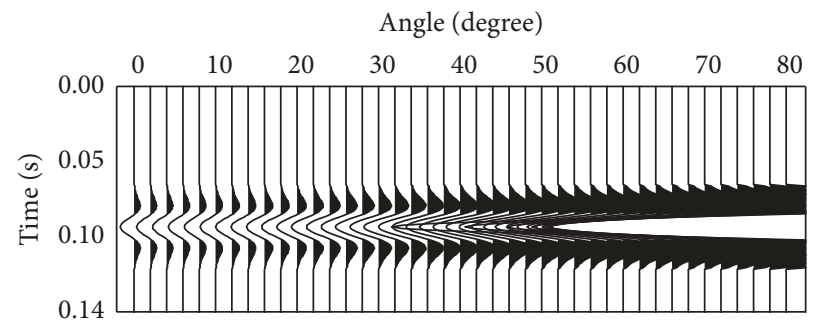

(c)

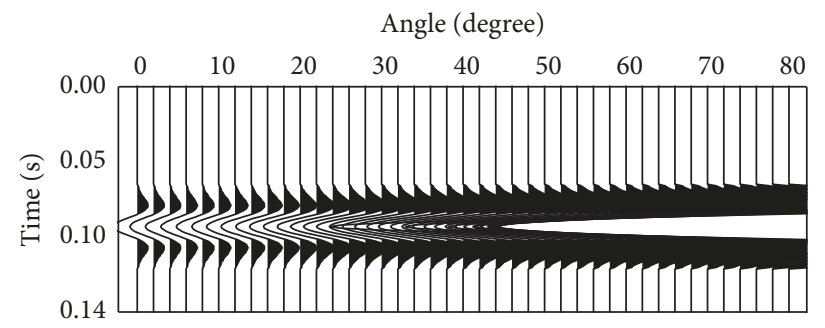

(e)

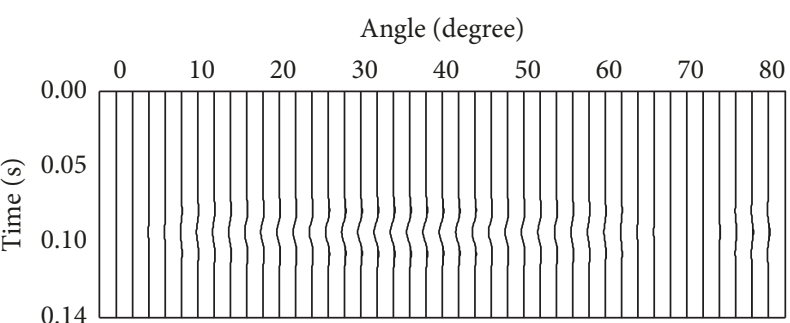

(b)

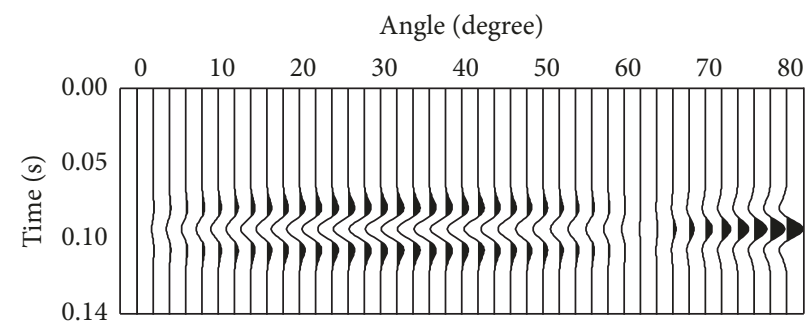

(d)

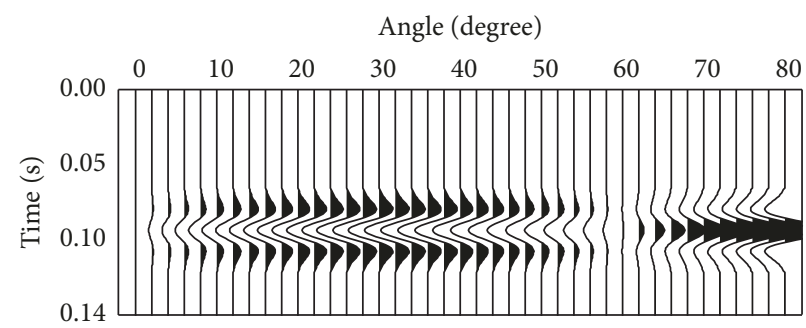

(f)

Figure 7: Synthesized AVA gathers of the VTI models with different $N / G$ ratios: (a) $N / G=0.1, P P$-wave; (b) $N / G=0.1, P S$-wave; (c) $N / G$ $=0.5, P P$-wave; (d) $N / G=0.5, P S$-wave; (e) $N / G=0.9, P P$-wave; (f) $N / G=0.9, P S$-wave.

increases sharply with increasing incidence angles, implying that anisotropy significantly influences $P P$-wave reflection coefficients. At a constant incidence angle, the differences of the $P P$-wave reflection coefficients first increase and then decrease with increasing $N / G$ ratios. The largest difference is observed at $N / G=0.5$. That is, when the thickness of the gas-saturated sand layer is half that of the finely layered reservoir, anisotropy has the greatest influence on the $P P$-wave AVA response. In Figure 6(b), the absolute values of the $P S$-wave differences increase, decrease to 0 , and continue to increase with increasing incidence angles. At incidence angles less than $45^{\circ}$, the differences in PS-wave reflection coefficients first increase and then decrease with increasing $N / G$ ratios. Similar to the $P P$-wave, the largest difference is observed at $N / G=0.5$. Overall, compared with the $P S$-wave, larger differences occur between the $P P$-wave reflection coefficients of models with and without anisotropy. Therefore, the AVA responses of $P P$-waves are more sensitive to anisotropy. Within intermediate incidence angles, large differences occur at higher incidence angles or the $N / G$ ratio close to 0.5 . That is, when the total thickness of gassaturated sand layers is equal to the thickness of shale layers in the finely layered reservoir, anisotropy has the greatest influence on the AVA responses. The influence of anisotropy on the AVA responses weakens with increases in the thickness difference between gas-saturated sand layers and shale layers.

3.3. AVA Gathers. We produce AVA gathers of the models shown in Table 3 with different $N / G$ ratios by the convolution of the Ricker wavelet [20-23] and reflection coefficients between the overburden and VTI medium. As the reflection coefficient curves change gradually with increasing $N / G$ ratios, we can deduce the law between AVA gathers and models with different $N / G$ ratios during the synthesis of the AVA gathers of $N / G=0.1, N / G=0.5$, and $N / G=0.9$ models.

The simulation results presented in Figures 7(a), 7(c), and $7(e)$ show that the $P P$-wave amplitudes increase with increasing incidence angles; abnormally large amplitudes of the $P P$ waves appear at near critical angles. As shown in Figures 7(b), $7(\mathrm{~d})$, and $7(\mathrm{e})$, the $P S$-wave amplitudes increase from 0 and then decrease to 0 with increasing incidence angles. Then, phase reversal occurs, and the amplitudes increase again with increasing incidence angles. The amplitudes of both the $P P$ and $P S$-waves are enhanced by the increase of $N / G$ ratios. Nevertheless, the phase reversal angles gradually decrease with increasing $N / G$ ratios. For the finely layered reservoir 
TABLE 4: Shale and oil-saturated sand layers parameters of model M7.

\begin{tabular}{lccccc}
\hline & $V_{P}(\mathrm{~m} / \mathrm{s})$ & $V_{S}(\mathrm{~m} / \mathrm{s})$ & Density $\left(\mathrm{g} / \mathrm{cm}^{3}\right)$ & Number of layers & Thickness $(\mathrm{m})$ \\
\hline Shale & 2250 & 900 & 2.2 & 64 & 0.7 \\
Sand & 2000 & 1400 & 2.1 & 65 & 0.7 \\
\hline
\end{tabular}

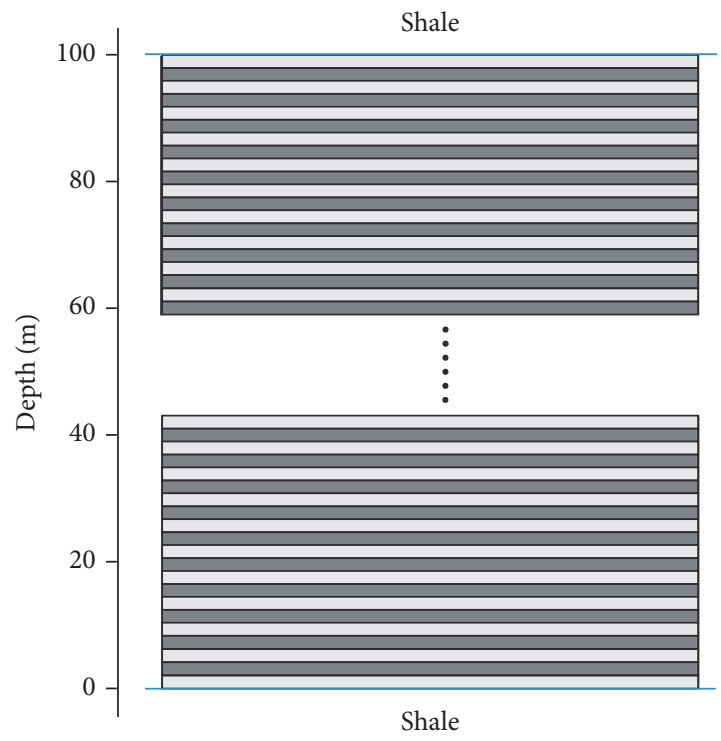

FIGURE 8: Schematic diagram of model M7.

model with different $N / G$ ratios, both the $P P$ - and $P S$-waves appear to have obviously abnormal AVA gathers. Thus, using $P P$ - and $P S$-waves simultaneously can help us to determine the $N / G$ ratios of finely layered reservoir and obtain the thickness of sand layers.

\section{Comparison with Stovas Model}

In this section, we compare our simulation results with those of Stovas et al. [9] to verify the reliability of our research. As shown in Figure 8, we adopt the same finely layered model (M7) proposed by Stovas et al. [9]. The model parameters are shown in Table 4 . The AVA gathers are computed by the same first derivation of the Gaussian wavelet with a central frequency of $40 \mathrm{~Hz}$ (Figure 9) as adopted by Stovas et al. [9]. In Figures 9(a) and 9(c), we consider every single thin shale or oil-saturated sand layer as an isotropic medium and calculate the reflection coefficients of the isotropic parts in every interface to derive the synthetic AVA gathers of the $P P$ - and PS-waves. Then, the total interbedded shale and oil-saturated sand layers are equivalent to a VTI model. As indicated by the blue lines in Figure 8, we only calculate the AVA reflections according to (8) at the top and bottom interfaces of model M7. The corresponding AVA gathers are shown in Figures 9(b) and 9(d).

The zero-angle $P P$-wave AVA reflection in Figure 9(a) is close to that in Figure 9(b). Therefore, it is reasonable to substitute the total interbedded shale and sand layers in model M7 with a VTI model, which is also confirmed by Stovas et al. [9]. In addition, we find some new rules based on our synthesized PP- and PS-wave AVA gathers. At small to intermediate incidence angles, the VTI model substitution is applicable to the finely layered reservoir because the disturbance energy between the top and bottom interfaces of model M7 is weak. However, the disturbance energy between the top and bottom interfaces of model M7 becomes stronger at large incidence angles, which is still acceptable. Overall, the $P P$ - and $P S$-wave AVA gathers of the finely layered model are similar to those of the VTI model (Figure 9). Thus, it is reasonable to use the VTI model to study the AVA responses of the finely layered reservoir.

\section{Conclusions}

In this work, we proposed a VTI model for finely layered reservoirs and studied the corresponding AVA responses. To describe the shale-sand interbedded structure, we adopted the $N / G$ theories for model parameter calculation. The theoretical model analyses show that the anisotropy of finely layered reservoir should not be ignored, especially for large incidence angles or total thicknesses of sand layers close to half that of the entire reservoir. At $N / G=0.5$, the anisotropy parameter $\varepsilon$ reaches the maximum and the anisotropy of the $P$-waves is the strongest. Furthermore, the AVA responses of the $P P$-waves were found to be more sensitive to anisotropy than those of $P S$-waves. The results of a comparative analysis of our model with that of Stovas et al. suggest that the VTI model substitution is appropriate and convenient for the study of AVA responses from the internal structural features of shale-sand layers in finely layered reservoirs. Our research will provide theoretical bases for the AVA inversion of finely layered reservoirs. 


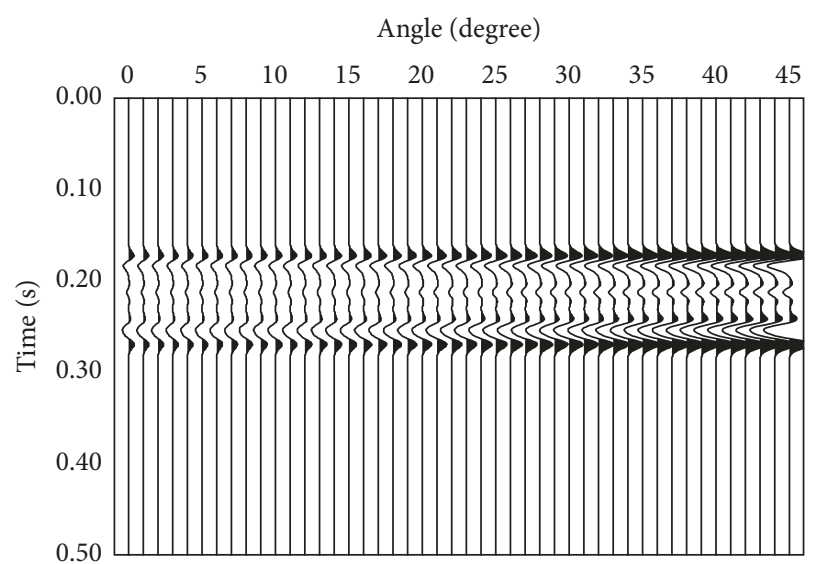

(a)

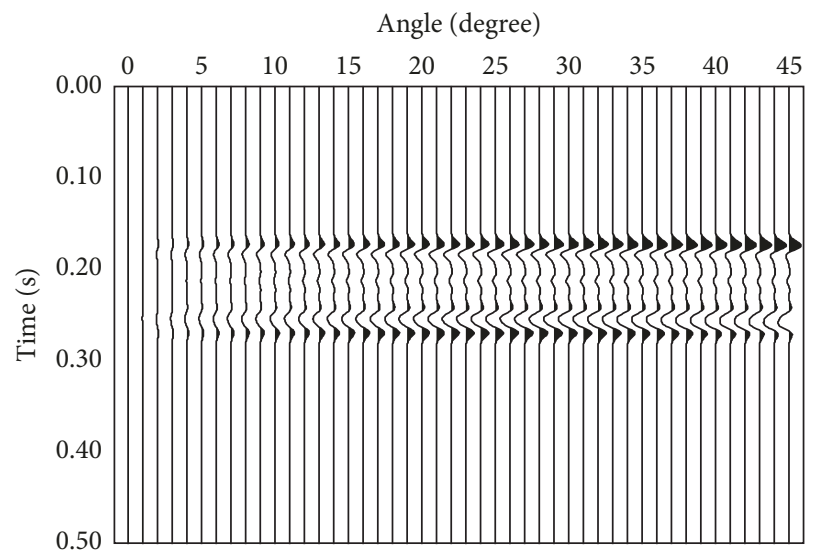

(c)

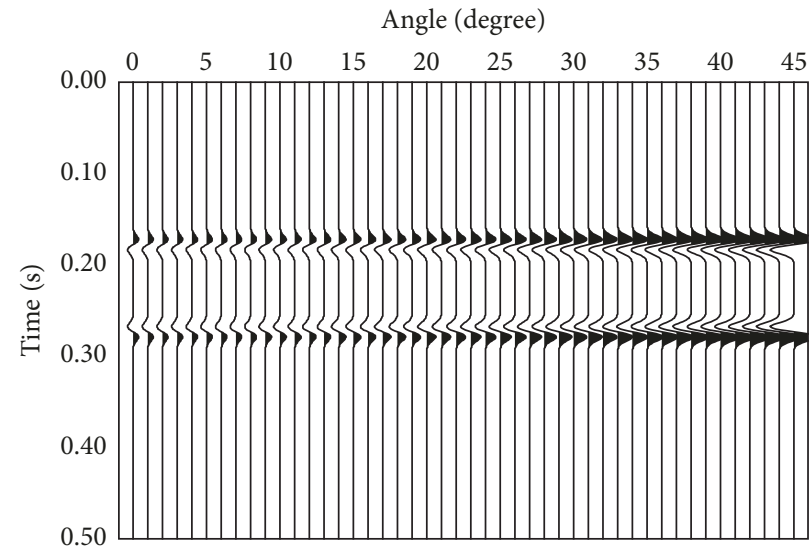

(b)

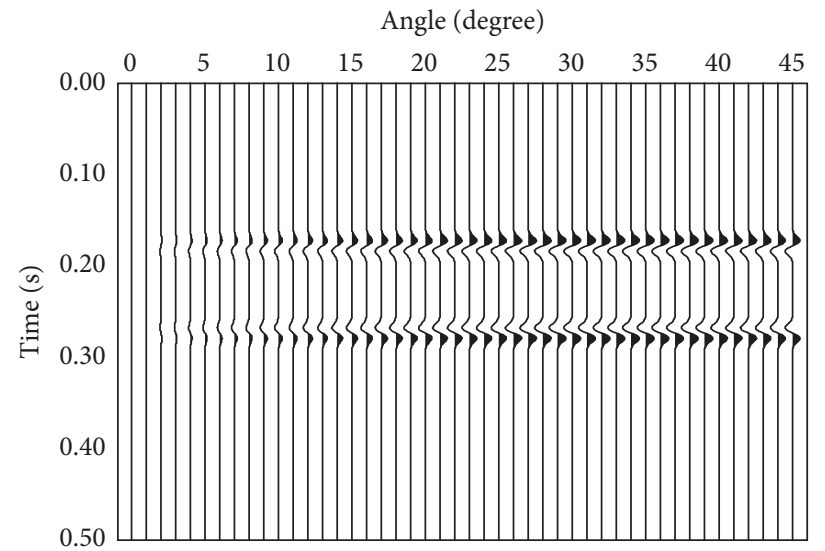

(d)

Figure 9: AVA gathers of model M7: (a) and (b) are PP-waves; (c) and (d) are PS-waves. In (a) and (c), the reflection coefficients of isotropic parts are calculated at each shale-sand interface. In (b) and (d), the total interbedded shale and sand layers are equivalent to a VTI model.

\section{Conflicts of Interest}

The authors declare that they have no conflicts of interest.

\section{Acknowledgments}

This research was primarily supported by the National Natural Science Foundation of China (41574126 and 41425017) and the Fundamental Research Funds for the Central Universities (2-9-2017-452).

\section{References}

[1] G. E. Backus, "Long-wave elastic anisotropy produced by horizontal layering," Journal of Geophysical Research: Atmospheres, vol. 67, no. 11, pp. 4427-4440, 1962.

[2] G. W. Postma, "Wave propagation in a stratified medium," Geophysics, vol. 20, no. 4, pp. 780-806, 1955.

[3] C. M. Sayers, "Long-wave seismic anisotropy of heterogeneous reservoirs," Geophysical Journal International, vol. 132, no. 3, pp. 667-673, 1998.

[4] R. Sidler and K. Holliger, "Seismic reflectivity of the sedimentcovered seafloor: effects of velocity gradients and fine-scale layering," Geophysical Journal International, vol. 181, no. 1, pp. 521-531, 2010.
[5] A. Stovas and B. Ursin, "Equivalent time-average and effective medium for periodic layers," Geophysical Prospecting, vol. 55, no. 6, pp. 871-882, 2007.

[6] D. A. G. Bruggeman, "Berechnung verschiedener physikalischer Konstanten von heterogenen Substanzen," Annalen der Physik, vol. 416, no. 8, pp. 665-679, 1935.

[7] T. Krey and K. Helbig, "A theorem concerning anisotropy of stratified media and its significance for reflection seismics," Geophysical Prospecting, vol. 4, no. 3, pp. 294-302, 1956.

[8] L. Vernik, D. Fisher, and S. Bahret, "Estimation of net-to-gross from $\mathrm{P}$ and S impedance in deepwater turbidites," The Leading Edge, vol. 21, no. 4, pp. 380-387, 2002.

[9] A. Stovas, M. Landrø, and P. Avseth, "AVO attribute inversion for finely layered reservoirs," Geophysics, vol. 71, no. 3, pp. C25C36, 2006.

[10] M. Schoenberg, "Reflection of elastic waves from periodically stratified media with interfacial slip," Geophysical Prospecting, vol. 31, no. 2, pp. 265-292, 1983.

[11] N. C. Banik, "An effective anisotropy parameter in transversely isotropic media," Geophysics, vol. 52, no. 12, pp. 1654-1664, 1987.

[12] L. Thomsen, "Weak elastic anisotropy", Geophysics, vol. 51, no. 10, pp. 1954-1966, 1986.

[13] A. Rüger, Reflection coefficients and azimuthal AVO analysis in anisotropic media [Ph.D. Thesis], Colorado School of Mines, Colorado, CO, USA, 1996. 
[14] A. Rüger, "P-wave reflection coefficients for transversely isotropic models with vertical and horizontal axis of symmetry," Geophysics, vol. 62, no. 3, pp. 713-722, 1997.

[15] Y. Liu and D. R. Schmitt, "Amplitude and AVO responses of a single thin bed," Geophysics, vol. 68, no. 4, pp. 1161-1168, 2003.

[16] W. Pan and K. A. Innanen, "AVO/AVF analysis of thin-bed in elastic media," in Proceedings of the SEG Houston 2013 Annual Meeting (SEG '13), pp. 373-377, Houston, TX, USA, September 2013.

[17] C. Yang, Y. Wang, and Y. H. Wang, "Reflection and transmission coefficients of a thin bed," Geophysics, vol. 81, no. 5, pp. N31-N39, 2016.

[18] K. Aki and P. Richards, Quantitative Seismology, University Science Books, Sausalito, Calif, USA, 2nd edition, 2002.

[19] F. Gassmann, “Über die Elastizität poroser Medien," Vierteljahrsschrift der Naturforschenden Gesellschaft in Zürich, vol. 96, pp. 1-23, 1951.

[20] N. Ricker, "The form and nature of seismic waves and the structure of seismograms," Geophysics, vol. 5, no. 4, pp. 348-366, 1940.

[21] N. Ricker, "Wavelet functions and their polynomials," Geophysics, vol. 9, no. 3, pp. 314-323, 1944.

[22] N. Ricker, "Wavelet contraction, wavelet expansion, and the control of seismic resolution," Geophysics, vol. 18, no. 4, pp. 769$792,1953$.

[23] N. Ricker, "The form and laws of propagation of seismic wavelets," Geophysics, vol. 18, no. 1, pp. 10-40, 1953. 


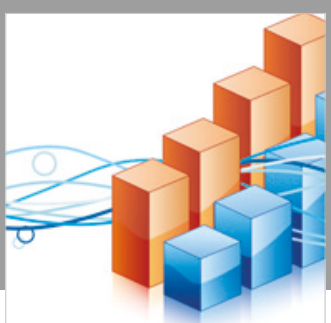

Advances in

Operations Research

\section{-n-m}
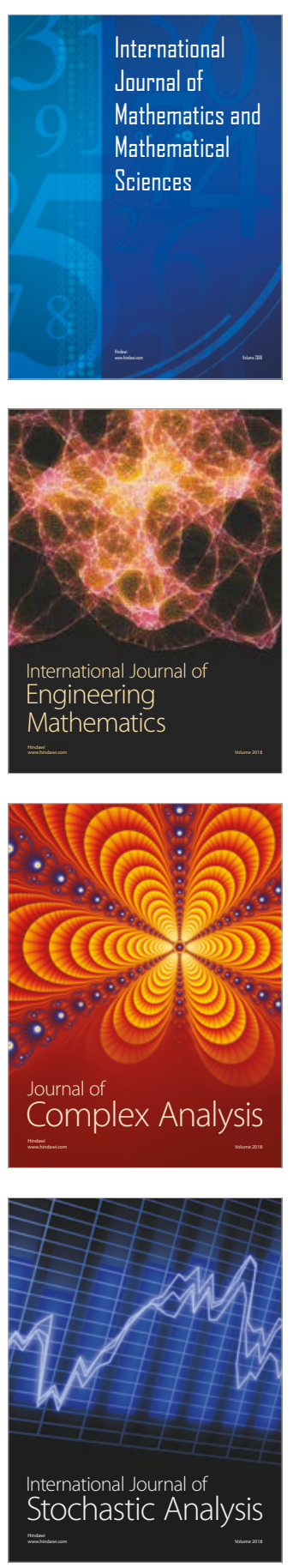
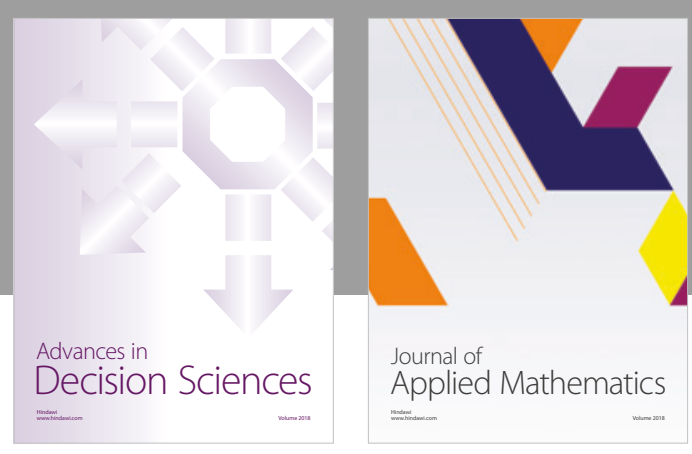

Journal of

Applied Mathematics
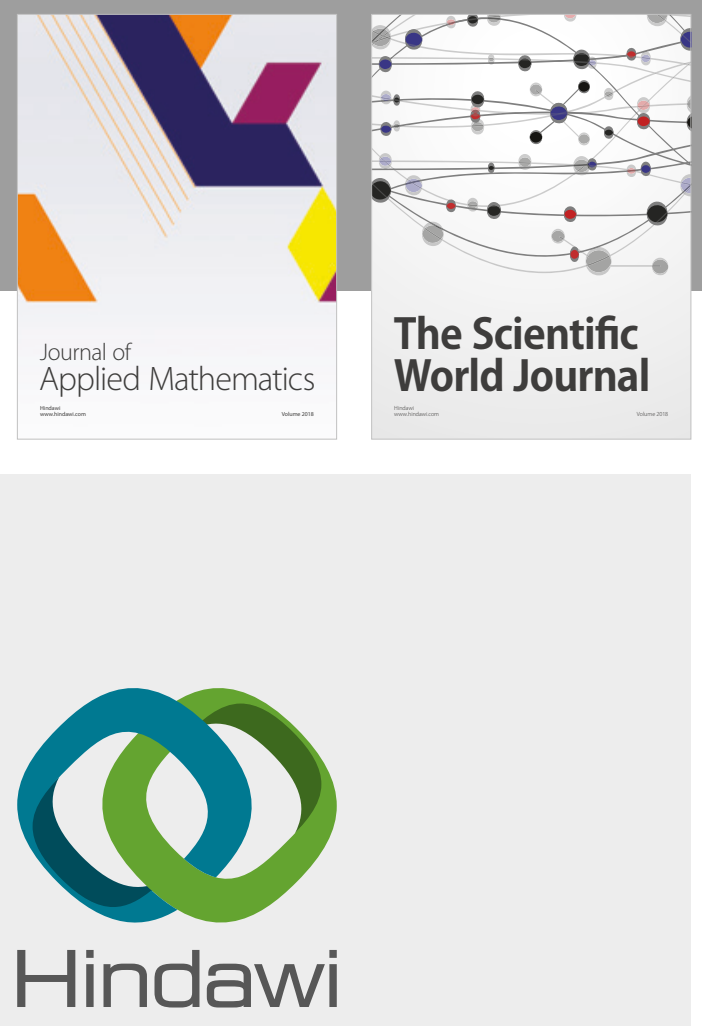

Submit your manuscripts at

www.hindawi.com

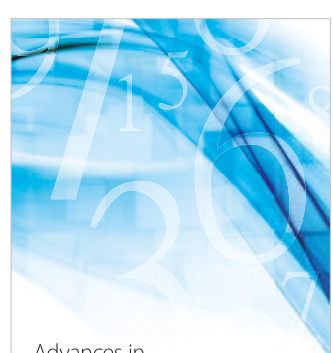

Advances in
Numerical Analysis
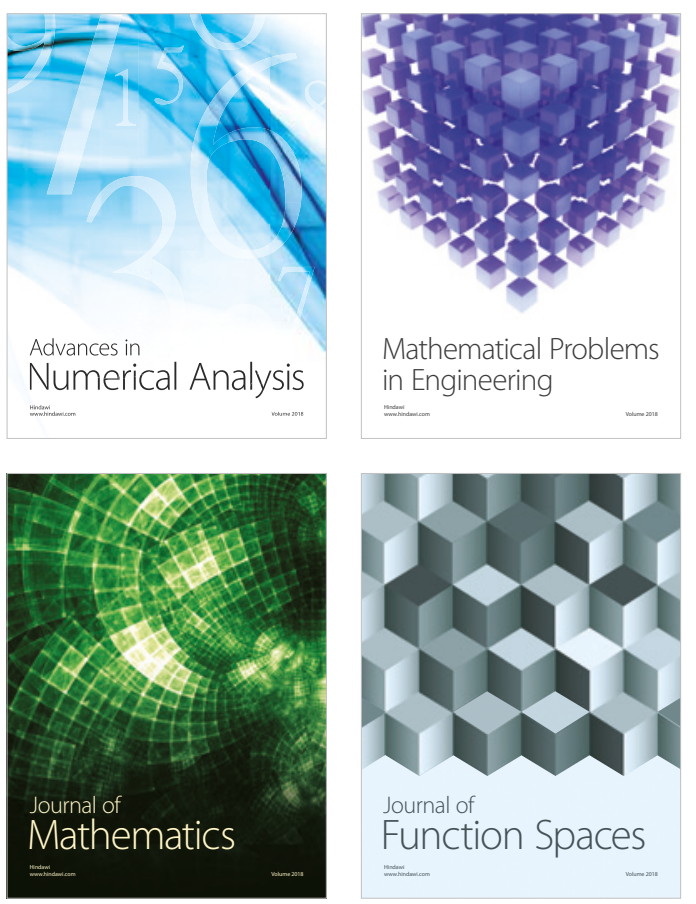

Mathematical Problems in Engineering

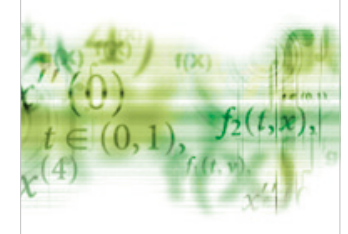

International Journal of

Differential Equations

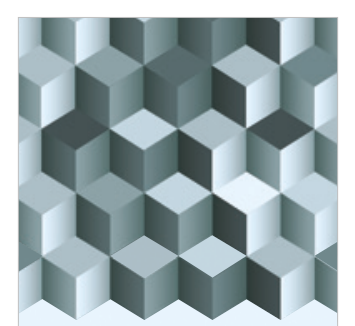

Journal of

Function Spaces

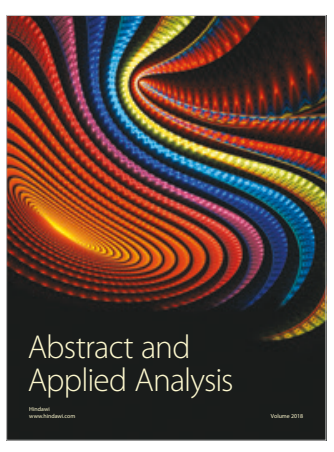

The Scientific

World Journal

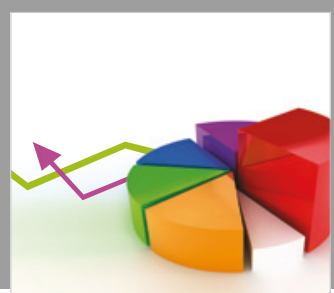

Journal of

Probability and Statistics
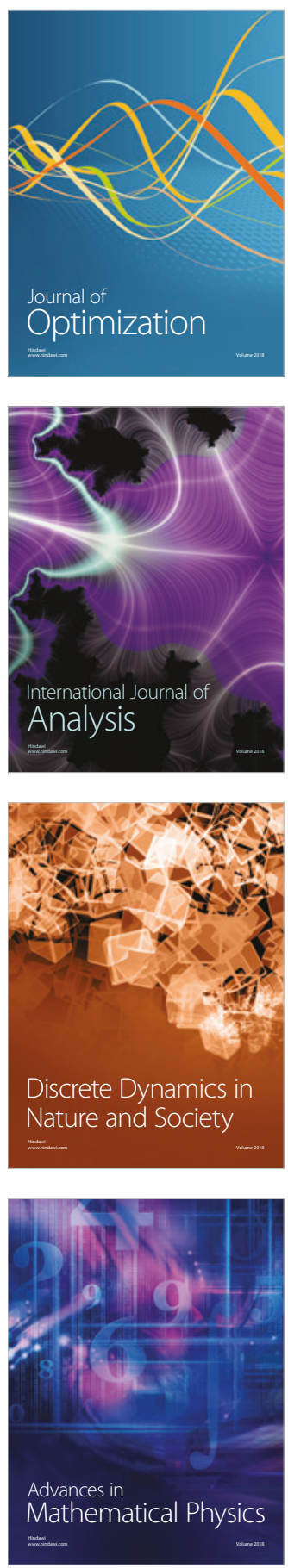